\title{
Vancomycin Resistance in ICU setting- A Rising Menace
}

\author{
Patel $\mathbf{U}^{1}$, Gedam DS ${ }^{2}$ \\ ${ }^{1}$ Dr. Umesh Patel, Associate Professor (Pediatrics), LN Medical College, Bhopal, MP, ${ }^{2}$ Dr. D Sharad Gedam, Professor, \\ Both are affiliated with Department of Pediatrics, LN Medical College, Bhopal, MP, India \\ Address for Correspondence: Dr Umesh Patel, Email: drumeshpatel@gmail.com
}

\begin{abstract}
Vancomycin-resistant organisms are significant nosocomial pathogens worldwide. Vancomycin resistant is a growing problem in the ICU setting. Treatment is difficult because of limited options (antibiotics) available.
\end{abstract}

Keywords: Vancomycin-resistant organism, Vancomycin resistance, Methicilline-resistant staphylococci

\section{Introduction}

Modern medicine has allowed doctors to treat disease aggressively. Some of these aggressive therapies are associated with prolong period use of profound immunosuppressive drugs and novel interventions. Few critically ill patients require prolong stay in ICU and hospital along with prolonged use of antibiotics. Patient in ICU frequently developed nosocomial, which are most of the time sever, difficult to treat and there is risk of recurrence. Risk of microbial colonization is again increase with multiple exposures of antimicrobial agents and use of immunosuppressant. Over the period of time these resistant organism become endemic in hospital environment, if medical care are not done properly. Methicilline-resistant staphylococci (both staphylococcus aureus-MRSA and coagulase-negative staphylococci) and Vancomycin resistant enterococcous (VER) are two most common and challenging organism [1]. Additional to these two Acinebactor baumannii, Pseudomonas aeruginosa and Stenotrophomonas maltrophilia are other infection that lead to unnecessary mortality and morbidity.

Vancomycin resistance, like many bacteria, can be spread from one person to another through physical contact or through contaminated objects.
Most often, infections are spread from the hands of health care workers to a patient in a hospital or other facility such as a nursing home. This infections are not usually spread through the air like the common cold or flu virus unless you have Vancomycin resistant pneumonia and are coughing, which is rare. Getting of infection in healthy person is unlikely. Infections typically occur among people who have weakened immune systems, such as people who have long-term illnesses or people who have had major surgery or other medical conditions; those have been treated with multiple antibiotics.

60 years back, soon after discovery, Vancomycin discarded from clinical use because of more toxic effect. There is greater interest in vancomycin now, 60 years after its discovery, than at any time in its history. The advent of pseudomembranous enterocolitis, coupled with the spread of methicillin-resistant Staphylococcus aureus, led to resurgence in the use of Vancomycin [2]. In 1980s, Vancomycin use again increase. With accelerated use, Vancomycin resistant Enterococcus (VRE) was reported in Europe (1986) and United State (1987) [3]. Till date six resistant patterns (Van A, Van B, Van C, Van D, Van E, Van G) have been reported [4]. These resistance can be spread from Enterococcus to other pathogens. 
Prevention and control of the spread of vancomycin resistance will require coordinated, concerted effort from various departments of the hospital, and can only be achieved if each of the following elements is addressed [5]: -

1. Prudent vancomycin use by clinicians

2. Education of hospital staff regarding the problem of vancomycin resistance

3. Early detection and prompt reporting of vancomycin resistance in enterococci and other gram positive microorganisms by the hospital microbiology laboratory

4. Immediate implementation of appropriate infection-control measures to prevent person-to-person transmission of VRE.

Verma $d$ et al in their study in northern India noticed high prevalence of aminoglycoside resistance in both categories of vancomycin sensitive and vancomycin resistant enterococci [6].

\section{Conclusion}

In last 2 decades, prevalence of hospital acquired antibiotic resistant pathogens increased significantly. In this situation hospital infection control program has increasing significance to control antibiotic resistant infections. Aggressive surveillance in asymptomatic patient admitted in
ICU and antibiotic control program to stop misuse of antimicrobial are important steps.

\section{References}

1. Cheah AL, Spelman T, Liew D et al. Enterococcal bacteraemia: factors influencing mortality, length of stay and costs ofhospitalization. Clin Microbiol Infect. 2013 Apr;19(4):E181-9. doi: 10.1111/1469-0691.12132. Epub 2013 Feb 7.

2. Levine DP. Vancomycin: A History. Clin Infect Dis. 2006 Jan 1;42 Suppl 1:S5-12.

3. Murray BE. Vancomycin-resistant enterococcal infections. N Engl J Med. 2000 Mar 9;342(10):710-21.

4. Depardieu F, Bonora MG, Reynolds PE, Courvalin P. The vanG glycopeptides resistance operon from Enterococcus faecalis revisited. Mol Microbiol. 2003 Nov;50(3):931-48.

5. Recommendations for preventing the spread of vancomycin resistance: Recommendations of the Hospital Infection Control Practices Advisory Committee (HICPAC). April 1995; 23(2):87-94.

6. Verma D, Sinha S, Ved Prakash. Prevalence and comparison of high-level aminoglycoside resistance in vancomycin sensitive and vancomycin resistant Enterococcus at a tertiary care hospital in Rohillkhand region. Int J Med Res Rev 2015;3(10):1162-1166. doi: 10.17511/ijmrr.2015.i10.210.

\section{How to cite this article?}

Patel U, Gedam DS. Vancomycin Resistance in ICU setting- A Rising Menace. Int J Med Res Rev 2015;3(10):11101111. doi: 10.17511/ijmrr.2015.i10.233. 